\title{
Perinatal Outcome and Frequency of Congenital Malformations in Pregnancy Complicated with Diabetes Type 1 and Type 2
}

\author{
Vjosa M. Kotori ${ }^{1,2}{ }^{*}$, Marina Ivanišević ${ }^{1}$, Josip Đelmiš ${ }^{1}$ \\ ${ }^{1}$ Department of Obstetrics and Gynecology, State Referral Centre for Diabetes in Pregnancy, School of \\ Medicine, Zagreb, Croatia \\ ${ }^{2}$ Currently at University Medical Centre of Kosovo, Prishtina, Kosovo \\ Email: vvjosak@gmail.com
}

Received 18 January 2016; accepted 21 February 2016; published 24 February 2016

Copyright $@ 2016$ by authors and Scientific Research Publishing Inc.

This work is licensed under the Creative Commons Attribution International License (CC BY). http://creativecommons.org/licenses/by/4.0/

(c) (i)

\section{Abstract}

Objectives: The objectives of this study were to evaluate and compare perinatal outcomes and frequency of congenital malformations in pregnancy that are complicated with type 1 and type 2 diabetes. Study Design: This prospective study included 557 pregnant women with type 1 diabetes and 149 pregnant women with type 2 diabetes that gave birth in the Clinic for Diabetes in Pregnancy in Zagreb, from January 2000 to December 2012. Results: Women with type 2 diabetes were significantly older than women with type 1 diabetes $(p<0.003 ; 32.8 \pm 5.5$ versus $29.3 \pm 3.2)$ and they have significantly higher BMI compared to type 1 diabetes $(P<0.001 ; 29.2 \pm 6.5$ versus $23.5 \pm$ 3.8). Comparing their delivery patterns, women with type 1 diabetes were significantly more likely to give birth by caesarean section than women with type 2 diabetes $(p<0.001 ; 466$ versus 82). The offspring of women with type 1 diabetes were more likely to be delivered preterm in comparison with offspring of women with type 2 diabetes (109 versus 31). Macrosomia is recognized characteristic of pregnancies complicated by diabetes and its incidence was significantly higher in women with type 1 diabetes compared to women with type 2 diabetes $(p<0.035 ; 174$ versus 38). Neonatal malformations were higher in women with type 1 vs. type 2 diabetes (12 versus 7), but not statistically significant. Glycosylated haemoglobin (HbA1c) levels were statistically significantly higher in the first trimester $(8.02 \%$ v. $6.72 \%)$, second $(7.55 \%$ versus $6.27 \%)$ and third trimester $(7.40 \%$ versus $6.03 \%)$ in women with type 1 and type 2 diabetes that gave birth to neonates with congenital malformations. Perinatal mortality was higher in pregnant women with type 1 diabetes than in women with type 2 diabetes ( 4 versus 2) but not statistically significant. Conclusion: Comparing perinatal mortality and frequency of congenital malformations

\footnotetext{
${ }^{*}$ Corresponding author.
} 
in women with type 1 and type 2 diabetes, we didn't find any important statistical differences. There is no significant difference in complication for babies of women with type 1 and type 2 diabetes, and it is likely that the most important causative factor is a high maternal blood glucose concentration.

\section{Keywords}

\section{Type 1 and Type 2 Diabetes Mellitus, Congenital Malformations, Macrosomia, Perinatal Mortality}

\section{Introduction}

Diabetes is the leading cause of death in the world and it is the leading cause of perinatal complications. Despite improvement in treatment strategies diabetes is still the main cause of blindness, kidney disease, amputation of extremities, heart disease and cerebro-vascular insult.

In the other site, the obesity pandemic is increasing problem worldwide to a pandemic proportion over the past 20 years. The increasing prevalence of obesity among females in reproductive age is of particular concern, too. Increasing trends of obesity is related with worldwide increase in the prevalence of type 2 diabetes. A worldwide increase in the prevalence of type 2 diabetes is now being realized in the pregnancy context with apparently similar or even worse outcomes to type 1 diabetes mellitus.

Since Sent Vincent Declaration in October 1989 that: the outcome achievement of pregnancy complicated with diabetes should be the same as non-complicated pregnancy [1], in the last years we see improvement of perinatal protection and decreased perinatal mortality. But, despite improved obstetric surveillance and better management of maternal hyperglycaemia over the last few decades, perinatal mortality and congenital malformations rates remains several fold higher in pregnancy complicated by diabetes than in the non-diabetic pregnancies [2].

Complication in diabetic pregnancy for mother are: preterm delivery, hypoglycaemia, ketoacidosis, urinary infection, traumatic delivery, preeclampsia and for foetus: abortion, IUGR, hypoxic-acidosis, traumatic delivery, congenital malformations and long term consequences of pregnancy and delivery.

Health (CEMACH) study it is shown similar results for both types of patients, while study of Balsells et al. and Clausen et al. have noted higher rates of perinatal morbidity and mortality, Taken together with previous investigations, there is no agreement regarding perinatal outcomes in women with type 1 and type 2 diabetes. In the Confidential Enquiry into Maternal and Child and congenital malformations in pregnancies complicated by type 2 diabetes than in those with type 1 diabetes. Therefore, further studies are required to compare pregnancy outcomes between women with type 1 and type 2 diabetes. Moreover, it is not known whether the factors related to obstetric and perinatal outcomes are similar for both types of diabetes in Croatia. Understanding these factors may help in preventing complications in pregnancy and in improving pregnancy outcomes in women with diabetes.

\section{The Aim}

The aim of our study was comparison of perinatal outcomes and frequency of congenital malformations in pregnancy complicated with type 1 and type 2 Diabetes Mellitus since the published data on foetal outcomes are still scarce.

\section{Methods}

This was a prospective study performed from January 2000 to December 2012. Subjects included in the study were identified by endocrinologist treating women with diabetes and during attendance at the specialist medical obstetric clinic to the completion of their pregnancy.

All women with type 1 and type 2 diabetes mellitus who delivered between January 2000 and December 2012 were recruited for the study.

The Diagnostic criteria used in this study were based on WHO recomandation for diagnosis of type 1 and type 2 diabetes. Here are the criteria: 
- Diabetes symptoms (e.g. polyuria, polydipsia and unexplained weight loss for Type 1) plus:

- Random venous plasma glucose concentration $\geq 11.1 \mathrm{mmol} / 1$ or

- Fasting plasma glucose concentration $\geq 7.0 \mathrm{mmol} / \mathrm{l}$ (whole blood $\geq 6.1 \mathrm{mmol} / \mathrm{l})$ or

- Two hour plasma glucose concentration $\geq 11.1 \mathrm{mmol} / 1$ two hours after $75 \mathrm{~g}$ anhydrous glucose in an oral glucose tolerance test (OGTT).

All data were prospectively collected and consist of the following categorical variables: age of pregnancy, BMI, weight gain during pregnancy, HbA1c during the first, second and third trimester, nephropathy, chronic hypertension, pregnancy outcomes (perinatal mortality, mode of delivery, preterm delivery), macrosomia and congenital malformations.

Glycaemic control was assessed by HbA1c, measured by high performance liquid chromatography (normal range 3.3\% - 5.7\%). Targets for glycaemic control were capillary blood glucose of $4.1-6.1 \mathrm{mmol} / \mathrm{l}$ pre-prandial, and two hour post-prandial glucose of $<7.5 \mathrm{mmol} / \mathrm{l}$ and the goal level of Hbalc were $\leq 6.5 \%$.

Women were treated with an intensive insulin regimen using four to five daily insulin injections a day or by continuo's subcutaneous infusion by an external pump and tested their blood glucose frequently-usually six to seven times a day.

Diabetic nephropathy was assessed on the basis of pre-pregnancy urinary albumin excretion rate and they were classified as absent (urinary albumin excretion rate $<30 \mathrm{mg} / 24 \mathrm{~h}$ ) incipient (30 - 300 mg/24h) or overt ( $>300 \mathrm{mg} / 24 \mathrm{~h}$ ). Chronic hypertension was defined as a systolic blood pressure level $\geq 140 \mathrm{mmHg}$ or a diastolic blood pressure level $\geq 90 \mathrm{mmHg}$.

Perinatal mortality comprised both foetal and neonatal deaths (foetal deaths were defined as still-births $\geq 22$ weeks of gestation or an infant weighing $\geq 500 \mathrm{~g}$ and neonatal death was defined as a death of a live born infant before the 28th day of life). Preterm delivery comprised delivery $<37$ weeks of gestation. Macrosomia was defined as a birth weight $>4000$ gr.

Data were analysed using SPSS Statistics 17. A p value, 0.05 was considered significant. Outcomes included newborn birth weight, Ponderal index, foetal macrosomia and the way of completion of pregnancy. Data were analysed with t tests, or X2-test.

\section{Results}

557 pregnant women with type $1 \mathrm{DM}$ and 149 pregnant women with type 2 DM have taken delivery in the Clinic for Diabetes in Pregnancy in Zagreb, from January 2000 to December 2012.

Women with type 1 diabetes gave birth to 563 newborns (4 twin pregnancies and 1 third) and 149 women with type 2 diabetes gave birth to 154 newborns (5 twin pregnancy).

In the period from y. 2000 to 2003 they were 19 (9.8\%) pregnant women with type 2 DM attending in our Clinic. Increasing number in the second period from y. 2004 to 2007 with 50 (24.6\%) women with type 2 and in the third period from y. 2008 to 2012 with 80 (25.9\%) was observed (see Table 1). The incidence of type 2 diabetes has increased in the general population and now affects many women of reproductive age. Thus the incidence of type 2 diabetes in pregnancy has increased.

Maternal data and their offspring's parameters are reported in Table 2.

Women with type 2 diabetes were significantly older than women with type 1 diabetes $(\mathrm{p}<0.003 ; 32.8 \pm 5.5$ versus $29.3 \pm 3.2)$ and they have significantly higher BMI than those with type 1 Diabetes $(\mathrm{p}<0.001 ; 29.2 \pm 6.5$ versus $23.5 \pm 3.8$ ) but the weight gain during pregnancy was significantly higher in women with type 1 Diabetes $(\mathrm{p}<0.003 ; 13.3 \pm 5.3$ versus $10.1 \pm 6.5)$.

Chronic hypertension was present in both groups, in 37 (6.5\%) pregnant women with type 1 diabetes from 557, and in 15 (9.8\%) pregnant women with type 2 diabetes from 149, and this difference didn't show any statistical significance. Diabetic nephropathy was present in 40 (12.6\%) pregnant women with type 1 diabetes.

Mean glycosylated haemoglobin values (HbA1c) for each trimester in both groups are shown in Table 2. In the first trimester, HbA1c was higher in pregnant women with type 1 diabetes then in pregnant women with type 2 diabetes $(\mathrm{p}<0.018 ; 7.42 \pm 1.6$ versus $7.4 \pm 1.4)$. In the second trimester HbA1c is drastically improved in women with type 1 and type 2 diabetes $(6.5 \pm 1.2$ versus $6.3 \pm 0.9)$ and in the third trimester $(6.6 \pm 1.3$ versus $6.6 \pm 1.2$ ) due to intensified treatment and control of diabetes.

There was no significant difference in Birth weight and Ponderal index between neonates born to mothers with type 1 diabetes and type 2 diabetes. Perinatal mortality was higher in pregnant women with type 1 diabetes then in women with type 2 diabetes but not statistically significant. 
Table 1. Number of pregnant women with diabetes type 1 and type 2 in three different periods.

\begin{tabular}{|c|c|c|c|c|c|}
\hline & & & \multicolumn{2}{|c|}{ Diagnose } & \multirow{2}{*}{ Total } \\
\hline & & & DM type II & DM type I & \\
\hline & & Number & 19 & 175 & 194 \\
\hline & 2000-2003 & \% Period & $9.8 \%$ & $90.2 \%$ & $100.0 \%$ \\
\hline & & $\%$ Total & $2.7 \%$ & $24.8 \%$ & $27.5 \%$ \\
\hline & & Number & 50 & 153 & 203 \\
\hline \multirow[t]{8}{*}{ Period } & 2004-2007 & \% Period & $24.6 \%$ & $75.4 \%$ & $100.0 \%$ \\
\hline & & $\%$ Total & $7.1 \%$ & $21.7 \%$ & $28.8 \%$ \\
\hline & & Number & 80 & 229 & 309 \\
\hline & 2008-2012 & \% Period & $25.9 \%$ & $74.1 \%$ & $100.0 \%$ \\
\hline & & $\%$ Total & $11.3 \%$ & $32.4 \%$ & $43.8 \%$ \\
\hline & \multirow{3}{*}{ Total } & Number & 149 & 557 & 706 \\
\hline & & \% Period & $21.1 \%$ & $78.9 \%$ & $100.0 \%$ \\
\hline & & $\%$ Total & $21.1 \%$ & $78.9 \%$ & $100.0 \%$ \\
\hline
\end{tabular}

Table 2. Parameters of pregnant women with DM-1 and DM-2 and their off springs.

\begin{tabular}{|c|c|c|c|}
\hline & $\mathrm{DM}-1(\mathrm{n}=557) \mathrm{X} \pm \mathrm{SD}$ & $\mathrm{DM}-2(\mathrm{n}=149) \mathrm{X} \pm \mathrm{SD}$ & $\mathrm{p}$ \\
\hline Age (year.) & $29.3 \pm 3.2$ & $32.8 \pm 5.5$ & $<0.003$ \\
\hline $\operatorname{BMI}\left(\mathrm{kg} / \mathrm{m}^{2}\right)$ & $23.5 \pm 3.8$ & $29.2 \pm 6.5$ & $<0.001$ \\
\hline Weight gain (kg) & $13.3 \pm 5.3$ & $10.1 \pm 6.5$ & $<0.003$ \\
\hline Chronic hypertension n (\%) & 37 (6.5\%) & $15(9.8 \%)$ & n.s. \\
\hline Diabetic nephropathy n (\%) & $40(12.6 \%)$ & $0(0 \%)$ & $<0.001$ \\
\hline HbA1c: first trimester (\%) & $7.42 \pm 1.6$ & $7.4 \pm 1.4$ & $<0.018$ \\
\hline HbA1c: second trimester (\%) & $6.5 \pm 1.2$ & $6.3 \pm 0.9$ & n.s. \\
\hline HbA1c: third trimester (\%) & $6.6 \pm 1.3$ & $6.6 \pm 1.2$ & n.s. \\
\hline Birth weight (g) & $3353.1 \pm 770$ & $3385 \pm 824.3$ & n.s. \\
\hline Ponderal index $\left(100 \mathrm{X} \mathrm{g} / \mathrm{cm}^{3}\right)$ & $2.82 \pm 0.3$ & $2.79 \pm 0.3$ & n.s. \\
\hline Perinatal mortality n (\%) & $4(0.7 \%)$ & $2(1.3 \%)$ & n.s. \\
\hline Caesarean section n (\%) & $466(93.5 \%)$ & $82(55.0 \%)$ & $<0.001$ \\
\hline Foetal macrosomia n (\%) & $174(31 \%)$ & $38(24.7 \%)$ & $<0.035$ \\
\hline Preterm delivery n (\%) & $109(19.7 \%)$ & $31(20.1 \%)$ & n.s. \\
\hline Neonatal malformations n (\%) & $12(2.2 \%)$ & $7(4.5 \%)$ & n.s. \\
\hline
\end{tabular}

Comparing their delivery patterns, women with type 1 diabetes were significantly more likely to be delivered by caesarean section then women with type 2 diabetes ( $p<0.001 ; 466$ versus 82 ). Thus, almost $45 \%$ of women with type 2 diabetes had a vaginal delivery. The offspring of women with type 1 diabetes were more likely to be delivered preterm in comparison with offspring of women with type 2 diabetes (109 versus 31). Macrosomia is well recognized characteristics of pregnancies complicated by diabetes and its incidence was significantly higher 
in women with type 1 diabetes compared to women with type 2 diabetes ( $\mathrm{p}<0.035$; 174 versus 38).

The frequency of neonatal malformations were higher in women with type $1 \mathrm{DM}$ compared with women with type 2 DM (12 versus 7), but not statistically significant.

Glycosylated haemoglobin (HbA1c) levels were statistically significantly higher in the first trimester in women with type 1 and type 2 DM that gave birth to neonates with congenital malformations (8.02\% v. 6.72.\%). In the second and third trimester HbA1c was lower than in first trimester but still significantly higher in women with diabetes that gave birth to neonates with congenital malformations (7.55\% versus $6.27 \%$ in second trimester and $7.40 \%$ versus $6.03 \%$ in third trimester-Table 3 below).

Congenital malformations were associated with poorer glycaemic control reflected through higher values of HbA1c.

\section{Discussion}

The introduction of preconception care, improvement in glycaemic control, better methods of foetal monitoring and sophisticated neonatal care have resulted in a dramatic improvement in foetal and maternal outcomes for women whose pregnancy is complicated by diabetes mellitus.

The great impact to maternal and foetal outcome has per conceptual glycaemic control, but despite this fact still around the half of women with type 1 diabetes plans their pregnancy and even the lower number of women with type 2 diabetes.

Data from Dublin clinics are similar with our finding that showed that pregnant women with type 2 diabetes tended to be heavier and older [3]. For that reason intensive life style support before pregnancy need to be addressed during preconception care especially for women with type 2 diabetes. Obesity per se carries his own risk for pregnant women. But, minimising unnecessary weight gain in obese subjects with type 2 diabetes can improve maternal glycaemic control, reduce the risk of macrosomia and improve pregnancy outcomes [4] as it is shown in our study. Weight gain during pregnancy in women with type 1 diabetes was significantly higher than in women with type 2 diabetes. Low glycaemic index food need to be introduced in nutrition therapy and fear for hypoglycaemia and its rate to be addressed to minimise unnecessary weight gain.

Clinical studies [5] [6] have shown that controlling glycaemia before conception reduces the occurrence of congenital malformations, which remain the leading cause of mortality and serious morbidity in infants of mother with diabetes mellitus. The period of organogenesis (the first trimester of pregnancy) is of great importance for the well development of foetus and hyperglycemia during this period remains a risk factor for the development of congenital anomalies. Based on that some adverse outcomes including foetal loss and major congenital anomaly, are related to glycaemic control before and during early pregnancy [7] [8]. Despite all this importance, in our study the first trimester of pregnancy shows a greater HbA1c in both groups of women with pregestational diabetes. This finding suggests the great role of preconception counselling for women with diabetes that intend to become pregnant and its good establishment must be in focus in our country to improve the outcomes of women with pregestational diabetes.

In addition to glycaemic control, we suggest that they are other metabolic factors important in determining pregnancy outcomes since our data shows that HbA1c in women with type 2 diabetes were slightly lower that in

Table 3. HbA1c in pregnant women with diabetes type 1 and type 2 that gave birth to neonates with congenital malformations.

\begin{tabular}{ccccc}
\hline & Congenital malformations & $\mathrm{N}$ & HbA1c & SD \\
\hline \multirow{2}{*}{$1^{\text {st }}$ trimester HbA1c (\%) } & No $^{*}$ & 687 & 6.72 & 1.58379 \\
& Yes $^{*}$ & 19 & 8.02 & 1.54119 \\
$2^{\text {nd }}$ trimester HbA1c (\%) & No $^{*}$ & 687 & 6.27 & 1.12515 \\
& Yes $^{*}$ & 19 & 7.55 & 1.90633 \\
$3^{\text {rd }}$ trimester HbA1c (\%) & No $^{*}$ & 687 & 6.03 & 1.2419 \\
& Yes $^{*}$ & 19 & 7.40 & 1.7369 \\
\hline
\end{tabular}

${ }^{*} \mathrm{p}<0.001$. 
those with type 1 diabetes but still pregnancy outcomes are poor. Risk for adverse outcomes are greater with poor glycaemic control (HbA1c $\geq 7.5 \%$ ) compared with fair HbA1c $<7.5 \%$ [6]. The gold standard level of HbA1c $<6.5 \%$ even with intensified treatment and post prandial glucose monitoring during pregnancy is hard to be achieved.

The recognition that congenital malformations were increased in the infant of diabetic mothers was first observed over 40 years ago and quickly linked with maternal hyperglycaemia [2]. Evolving data suggest that the risk of serious adverse outcomes including congenital malformations and perinatal mortality is similar to or increased in type 2 diabetes compared with type 1 diabetes [9]. A recently published meta-analyses including 33 studies of 7966 pregnancies in type 1 diabetes and 3781 pregnancies in type 2 diabetes showed that women with type 2 diabetes had a significantly higher risk of perinatal mortality but no significant difference in rates of malformations [10].

Caesarean section rates for women with pregestational diabetes in most parts of the world are over $50 \%$ [2]. The indication for Caesarean section are often multiple and vary with individual hospital policy. The rate tends to be lower in women with type 2 diabetes. For the obstetrician the major consideration influencing the mode of delivery remains the risk of birth injury [2]. The caesarean section maybe related to macrosomia, which is greater in type 1 Diabetes [2] but they might be other factors that influence obstetrician decision for this mode of delivery which was very high in our study in comparison with other studies. Pregnant women with type 1 and type 2 diabetes have increased rates of spontaneous abortions, hypertensive disorders, preterm delivery and caesarean deliveries while their foetuses are at risk for congenital anomalies [7] [11].

As in type 1 diabetes, there is a high risk of foetal mortality and morbidity associated with type 2 diabetes. In our study we didn't find any significant difference in perinatal mortality and neonatal malformations between type 1 and type 2 diabetic women. Some data indicate that the risk of an adverse pregnancy outcome in women with type 2 diabetes may be even greater than in women with type 1 diabetes. A retrospective analysis of data from 61 consecutive pregnancies in women with type 2 diabetes by Clansen et al. [12] in Copenhagen reported that perinatal mortality was increased 4 and 9 fold in patients with type 2 diabetes compared with those with type 1 diabetes. The rate of major congenital malformations was more than double that in the type 1 diabetes population [12]. Consistent with these findings, a recent review by Kinsley et al. [10] from the Dublin Diabetes in pregnancy service, compared pregnancy outcomes in women with type 2 diabetes $(n=56)$ versus pregnancy outcomes in women with type 1 diabetes $(\mathrm{n}=372)$. Women with type 2 diabetes had increased rate of perinatal mortality and lethal congenital malformations compared with women with type 1 diabetes. Some other studies have demonstrated elevated perinatal mortality rate as well as obstetric complications among women with type 2 diabetes in pregnancy [13] [14].

Metabolic disturbances related to glucose intolerance and hyperglycaemia can impair the development of foetuses and newborns. Epidemiological studies have reported that poorly controlled debates in pregnant women with hyperglycaemia in early pregnancy is a risk factor for major congenital anomalies and poor pregnancies outcomes, and data suggest that the better maternal metabolic control is in early foetal development, less anomaly rate will be developed. It is of great importance to establish good preconception counselling for women with diabetes who intend to become pregnant. There is evidence that this improves the outcomes in women with pregestational diabetes [7].

There is considerable evidence that the risk of foetal loss and malformations in diabetic pregnancy is related to the severity of hyperglycaemia, as reflected by the HbA1c during the first trimester for patients with ether type 1 or type 2 diabetes [7] [15] [16]. Ideally, Hba1c should be within the normal range (usually 4.0\% - 6.0\%) by the time of conception [16].

The present study has certain limitations that must be taken into account when interpreting the data. First limitations include a missing pre-conception HbA1c data in women with Type 1 and Type 2 diabetes and second missing the rate of hypoglycemic events in women with type 1 diabetes in the first trimester that might influence HbA1c.

\section{Conclusions}

Comparing perinatal mortality and frequency of congenital malformations in women with type 1 and type 2 diabetes, we didn't find any important statistical differences. There is no significant difference in complication for babies of women with type 1 and type 2 diabetes, and it is likely that the most important causative factor is a 
high maternal blood glucose concentration. Like in the other studies it is shown that improvement in outcome for the baby can be achieved through active management of the mother's diabetes.

In generally, Women with diabetes who are at reproductive age should be identified as members of a high risk group. Access to specialized pre-pregnancy clinics should be made available where their diabetes can be managed throughout pregnancy by a multidisciplinary team.

Risks from adverse pregnancy outcomes may be reduced to minimum by adequate preconceptions counselling of diabetic patients. So, the most challenging aspect remains pre-conception blood glucose control avoiding peri-conception hyperglycaemia [17].

\section{References}

[1] St. Vincent Declaration. A Model for Prevention and Self-Care. 10-12 October 1989; St. Vincent, Italy. (A Meeting Organised by the World Health Organisation and the International Diabetes Federation.) https://www.idf.org/sites/default/files/attachments/2009_2_Hall_Felton.pdf

[2] McCance, D.R. (2011) Pregnancy and Diabetes. Best Practice \& Research Clinic Endocrinology \& Metabolism, 25, 945-958. http://dx.doi.org/10.1016/j.beem.2011.07.009

[3] Kinsley, B.T., Firth, R.G., Byrne, M.M., et al. (2006) Intensive Management of Pregestational Diabetes Mellitus in Pregnancy: Comparison Outcome of Type 2 Diabetes with Type 1 Diabetes. Diabetologia, 49, 67. Abstract, A0106.

[4] Nohr, E.A., Bech, B.H. and Vaeth, M. (2007) Obesity, Gestational Weight and Preterm Birth: A Study within the Danish National Birth Cohort. Paediatric and Perinatal Epidemiology, 21, 5-14.

[5] The Diabetes Control Complications Trial Research Group (1996) Pregnancy Outcomes in the Diabetes Control and Complications Trial. American Journal of Obstetrics \& Gynecology, 174, 1343-1353. http://dx.doi.org/10.1016/S0002-9378(96)70683-X

[6] Temple, R. Aldridge, V., Greenwood, R., et al. (2002) Association between Outcome of Pregnancy and Glycemic Control in Early Pregnancy in Type 1 Diabetes: A Population Based Study. BMJ, 325, 1275-1276. http://dx.doi.org/10.1136/bmj.325.7375.1275

[7] Ray, J.G., O’Brien, T.E. and Chan, W.S. (2001) Preconception Care and the Risk of Congenital Anomalies in the Offspring of Women with Diabetes Mellitus. A Meta-Analysis. QJM, 94, 435-444.

[8] Mills, J.L., Simpson, J.L., Driscoll, S.G., et al. (1988) Incidence of Spontaneous Abortion among Normal Women and Insulin Dependent Diabetic Women Whose Pregnancies Were Identified within 21 Days of Conceptions. The New England Journal of Medicine, 319, 1617-1623. http://dx.doi.org/10.1056/NEJM198812223192501

[9] Temple, R.C. and Murphy, H. (2010) Type 2 Diabetes-An Increasing Problem. Journal of Clinical Endocrinology and Metabolism, 24, 591-604.

[10] Balsells, M., Garcia-Patterson, A., Gich, I., et al. (2009) Maternal and Fetal Outcome in Women with Type 2 versus Type 1 Diabetes Mellitus: A Systematic Review and Metaanalysis. The Journal of Clinical Endocrinology \& Metabolism, 94, 4284-4291. http://dx.doi.org/10.1210/jc.2009-1231

[11] Confidential Enquiry into Maternal and Child Health (CEMACH) (2005) Pregnancy in Women with Type 1 and Type 2 Diabetes, 2002-2003. CEMACH, England, Wales and Northern Ireland, London, United Kingdom.

[12] Clausen, T.D., Mathiesen, E., Ekborn, P., et al. (2005) Poor Pregnancy Outcome in Women with Type 2 Diabetes. Diabetes Care, 28, 323-328. http://dx.doi.org/10.2337/diacare.28.2.323

[13] McElduff, A., Ross, G.P., Langstrom, J.A., et al. (2005) Pregestational Diabetes and Pregnancy. An Australian Experience. Diabetes Care, 28, 1260-1261. http://dx.doi.org/10.2337/diacare.28.5.1260

[14] Cheung, N.W., McElduff, A. and Ross, G.P. (2005) Type 2 Diabetes in Pregnancy. A Wolf in Sheep’s Clothing. Australian \& New Zealand Journal of Obstetrics \& Gynaecology, 45, 479-483. http://dx.doi.org/10.1111/j.1479-828X.2005.00480.x

[15] American Diabetes Association (2004) Preconception Care of Women with Diabetes. Diabetes Care, 27, S76-S78. http://dx.doi.org/10.2337/diacare.27.2007.S76

[16] Gunton, J.E., Morris, J., Boyce, S., et al. (2002) Outcome of Pregnancy Complicated by Pregestational Diabetes-Improvement in Outcomes. Australian \& New Zealand Journal of Obstetrics \& Gynaecology, 42, 478-481. http://dx.doi.org/10.1111/j.0004-8666.2002.00478.x

[17] Michaele Weindling, A. (2009) Offspring of Diabetic Pregnancy: Short Term Outcomes. Seminars in Fetal \& Neonatal Medicine, 14, 111-118. 